\title{
Atomic Force Microscopy
}

National Cancer Institute

\section{Source}

National Cancer Institute. Atomic Force Microscopy. NCI Thesaurus. Code C17754.

A technique utilizing a scanning probe to image and analyze the surface of a material with atomic-level resolution. 